\title{
Triad3a induces the degradation of early necrosome to limit RipK1-dependent cytokine production and necroptosis
}

\author{
Norah A. Alturki ${ }^{1,2}$, Scott McComb ${ }^{3}$, Ardeshir Ariana ${ }^{1}$, Dikchha Rijal ${ }^{1}$, Robert G. Korneluk ${ }^{1,4}$, Shao-Cong Sun ${ }^{5}$, \\ Emad Alnemri ${ }^{6}$ and Subash Sad ${ }^{1,7}$
}

\begin{abstract}
Understanding the molecular signaling in programmed cell death is vital to a practical understanding of inflammation and immune cell function. Here we identify a previously unrecognized mechanism that functions to downregulate the necrosome, a central signaling complex involved in inflammation and necroptosis. We show that RipK1 associates with RipK3 in an early necrosome, independent of RipK3 phosphorylation and MLKL-induced necroptotic death. We find that formation of the early necrosome activates K48-ubiquitin-dependent proteasomal degradation of RipK1, Caspase8, and other necrosomal proteins. Our results reveal that the E3-ubiquitin ligase Triad3a promotes this negative feedback loop independently of typical RipK1 ubiquitin editing enzymes, CIAPs, A20, or CYLD. Finally, we show that Triad3a-dependent necrosomal degradation limits necroptosis and production of inflammatory cytokines. These results reveal a new mechanism of shutting off necrosome signaling and may pave the way to new strategies for therapeutic manipulation of inflammatory responses.
\end{abstract}

\section{Introduction}

Macrophage-like cells are present throughout the body and play a major role in initiating inflammatory responses to control pathogens ${ }^{1-4}$. TLR signaling induces MyD88dependent and TRIF-dependent signaling, which leads to the production of cytokines and chemokines ${ }^{5-8}$, and recruitment of myeloid cells ${ }^{9}$.

Apoptosis promotes cell death during embryogenesis and in the elimination of self-reactive cells ${ }^{10,11}$. Cell death that occurs during infections proceeds through parallel cell death programs ${ }^{12}$. In contrast to apoptosis, inflammatory cell death results in cell-rupture and release of intracellular contents including cytokines and numerous danger-associated molecular patterns (DAMPs) to the

Correspondence: Subash Sad (subash.sad@uottawa.ca)

'Department of Biochemistry, Microbiology, and Immunology, Faculty of Medicine, University of Ottawa, Ottawa, Canada

${ }^{2}$ Applied Medical science, King Saud University, Riyadh, Saudi Arabia

Full list of author information is available at the end of the article.

Edited by G. Raschellà external milieu, which induces systemic amplification of inflammation ${ }^{10,13,14}$. Inflammatory cell death by pathogens is now considered to be a key driver of pathogen virulence ${ }^{15-19}$.

While necrosis was once considered to be an accidental, uncontrolled mode of inflammatory cell death, a pathway of regulated necrosis, called "necroptosis" is now known to be induced by TNF-R or IFN-IR engagement ${ }^{14,17,20-25}$. Necrosome signaling involves RipK1-FADD-Caspase-8 interaction, which leads to the phosphorylation of RipK $3^{26-28}$. Activated RipK3 phosphorylates mixed lineage kinase domain-like protein (MLKL), resulting in trimerization of MLKL and its relocation to the cell membrane, causing membrane rupture and necroptosis ${ }^{29}$. We have revealed a novel mechanism of proteasomal degradation of RipK1 and other interacting proteins by Triad3a. Our results indicate that during early necrosome signaling, Triad3a mediates the degradation of RipK3 interacting proteins to regulate necroptosis and expression of inflammatory cytokines.

\section{(c) The Author(s) 2018}

(c) (i) Open Access This article is licensed under a Creative Commons Attribution 4.0 International License, which permits use, sharing, adaptation, distribution and reproduction cc) in any medium or format, as long as you give appropriate credit to the original author(s) and the source, provide a link to the Creative Commons license, and indicate if changes were made. The images or other third party material in this article are included in the article's Creative Commons license, unless indicated otherwise in a credit line to the material. If material is not included in the article's Creative Commons license and your intended use is not permitted by statutory regulation or exceeds the permitted use, you will need to obtain permission directly from the copyright holder. To view a copy of this license, visit http://creativecommons.org/licenses/by/4.0/. 


\section{Results}

Necrosome activation leads to the degradation of various interacting proteins

Combined TLR4 stimulation (by LPS) and caspase inhibition (by zVAD) in macrophages causes the phosphorylation of RipK1 and RipK3, which can be observed as a slightly slower migrating protein band in western blot. Consistent with this, RipK1 and RipK3 were resolved as a single band upon dephosphorylation by calf intestinal phosphatase (CIP) (Fig. 1a). Coincident with phosphorylation of RipK3, we noted a progressive reduction in the levels of RipK1 after $2 \mathrm{~h}$ post stimulation (Fig. 1a). Treatment of macrophages with zVAD in the absence of any additional stimulation did not induce the phosphorylation of RipK1 or RipK3, and failed to have any impact on the levels of RipK1 (Fig. 1b). Stimulation of cells with LPS in the absence of zVAD, induced the phosphorylation of RipK1, but not RipK3, without having any impact on the levels of RipK1 (Fig. 1c). Thus, consistent with previous results ${ }^{30}$, combined LPS and $\mathrm{zVAD}$ was required for the phosphorylation of RipK3 that correlated with progressive disappearance of RipK1 and eventual cell death by necroptosis (Fig. 1c-e and Fig. S1 A, B). We also measured the expression of protein phosphatase-2, B subunit, a ubiquitous phosphatase in eukaryotic cells, and observed that this protein is also not degraded necrosome signaling (Fig. S1 C). Progressive loss of RipK1 was also observed when cells were stimulated with zVAD in the presence of highly reduced amount of LPS (Fig. S1 D). Reduction in the levels of RipK1 following necroptotic stimulus was not due to poor transcription of RipK1 as measured by qRT-PCR (Fig. 1f).

Although the reduction in RipK1 levels correlated with phosphorylation of RipK3 (Fig. 1d), the relative levels of RipK3 did not diminish during early necrosome signaling, and no cleavage bands of RipK1 or RipK3 were observed (Fig. S1 A, B). Loss of RipK1 was also noted when necroptosis was induced with TNF $\alpha+z V A D$ (Fig. 1g-i), and again correlated with the timing of RipK3 phosphorylation (Fig. 1g, h). Many other components of the necrosome were degraded following LPS + zVAD stimulation, including Caspase-8 (Casp-8), FADD, TRAF2, and cIAP-1 and -2 (Fig. 1j, k). In contrast, there was an increase in the expression of RipK3, CYLD, and A20 following stimulation of cells with LPS + zVAD (Fig. 1j, $\mathrm{k}$ ), indicating that all proteins are not degraded during necrosome signaling.

Reduction in the levels of Casp-8 following necroptotic stimulus was not due to poor transcription of Casp-8 (Fig. S1 E). Substitution of the pan-caspase inhibitor (zVAD) with the Casp-8 inhibitor (zIETD) failed to induce the loss of RipK1 expression or necroptosis of macrophages (Fig. S1 F, G). While Casp-8 inhibitor reduced the Casp- 8 activity to baseline levels
(Fig. S1 H), it did not rescue the loss of RipK1 expression following stimulation by LPS + zVAD (Fig. S1 I). This suggests that the loss of RipK1 expression is not due to cleavage of RipK1 by Casp-8. Furthermore, Casp8 itself was degraded (Fig. 1j).

\section{Necrosomal degradation is driven by RipK3}

The canonical kinase function of RipK1 is mediated by the catalytic triad that comprises K45-E63-D156 ${ }^{31}$. The two critical kinase regions, K45 and D138 complement each other in driving necrosome signaling and inflammatory responses ${ }^{32,33}$. We next tested whether the K45 kinase region of RipK1, which has been shown to promote necroptosis under some conditions ${ }^{19}$, was required for the degradation of RipK1. K45A mutation of RipK1 resulted in slightly reduced RipK3 phosphorylation and necroptosis but failed to have any significant impact on the degradation of RipK1 (Fig. 2a, b). In contrast, the degradation of RipK1 and necroptosis was dependent on the K45 region of RipK1 when cells were stimulated with TNF $\alpha+z V A D$ (Fig. S2 A, B). Following LPS/zVADinduced necroptosis, the reduction in the levels of RipK1, Casp-8, cIAP1,2, and FADD was dependent on TRIF, but not on Myd88 signaling (Fig. 2c, d), whereas for TNF $\alpha+$ zVAD stimulation, degradation of RipK1 and necroptosis were not dependent on TRIF (Fig. S2 C, D). Overall, we observed a strong correlation between RipK1 degradation, RipK3 phosphorylation, and eventual progression to necroptotic cell death, and a significant difference in the mechanism of RipK1 degradation following TNF- $\alpha$ - or LPS-induced necrosome signaling.

RipK3-deficient cells were protected against necroptosis and there was no degradation of RipK $1, \operatorname{cIAP} 1 / 2$, and Caspase-8 (Fig. 2e, f and Fig. S2 E). In contrast, when cells were stimulated with LPS only, which does not induced necroptosis, RipK3 did not have any impact on the expression of RipK1, cIAP1/2, and Casp-8 (Fig. S2 F). We wondered about a possible role for the downstream mediator of necroptotic cell death, MLKL ${ }^{29}$. Degradation of RipK1 and Casp-8 was not inhibited in MLKL-deficient macrophages (Fig. 2g and Fig. S2 G). As expected, MLKLdeficient macrophages were resistant to necroptotic cell death (Fig. 2f). We evaluated the expression of these proteins in $\operatorname{Irf9^{-/-}}$ and Ifnar1 $1^{-/-}$macrophages that are resistant to necroptosis ${ }^{30}$. Our results indicate that the degradation of these proteins was not modulated in $\operatorname{Irf} 9^{-1}$ - and Ifnar ${ }^{-/-}$macrophages (Fig. S2 H, I), suggesting that degradation continues during necrosome signaling in the absence of cell death. Loss of MLKL led to a significant decrease in RipK3 phosphorylation, suggesting the presence of a feedback role for MLKL in RipK3 phosphorylation (Fig. 2g). RipK3 was also required for necroptosis and the degradation of RipK1 following stimulation of cells with TNF $\alpha+z V A D$ (Fig. 2h, i). Thus, 


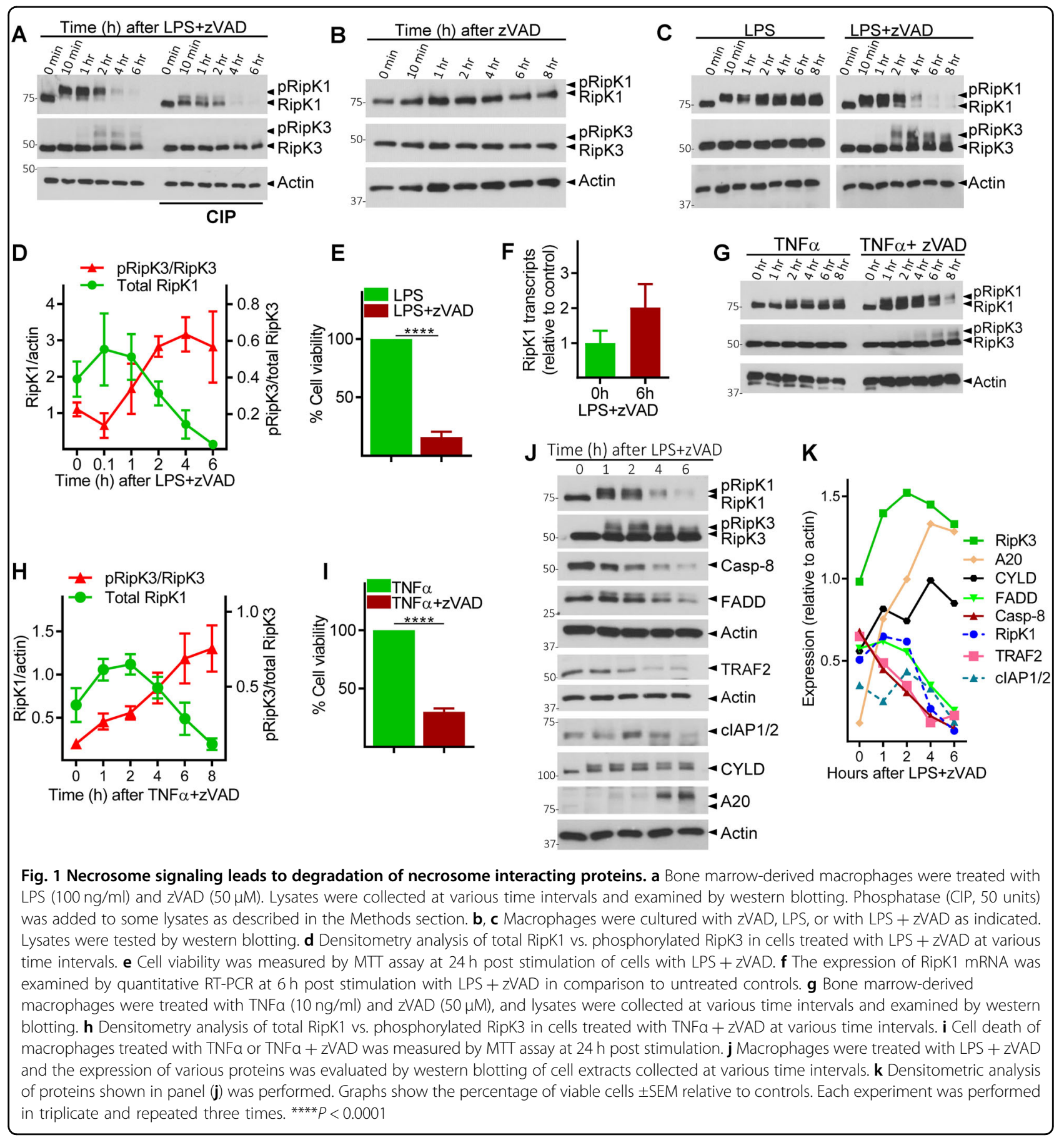

RipK3, but not MLKL is required for necrosomal degradation in macrophages following necroptotic stimulus.

\section{Necrosomal degradation is driven by early RipK1-RipK3 interaction}

Nec-1 has been shown to bind the kinase domain of RipK1 between the $\mathrm{N}$ - and C-lobes near the activation loop $^{34}$, and lock RipK1 in an inactive conformation ${ }^{34}$.
Nec-1 completely abrogated the phosphorylation of RipK3, degradation of RipK1 and cell death by necroptosis (Fig. 3a, c, d). Similar results were obtained with Nec-1S (Fig. S3 A, B), a more specific inhibitor of RipK $1^{35}$. Nec-1 had no impact on the phosphorylation of RipK1 (Fig. 3a). Inhibition of the p38MAPK resulted in potent inhibition of RipK1 phosphorylation but had no impact on the reduction of RipK1 levels (Fig. S3 C). Despite an obvious 

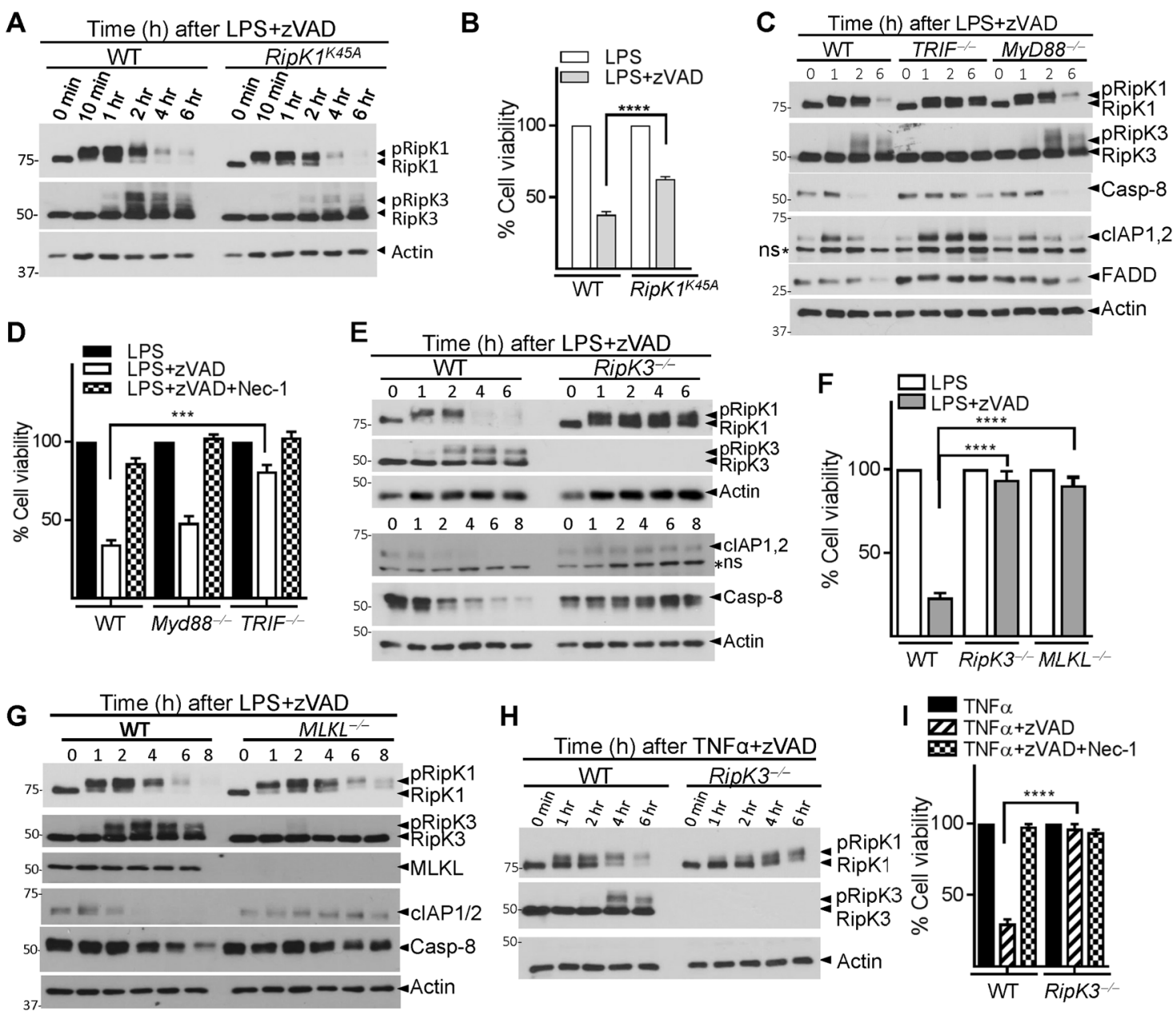

H

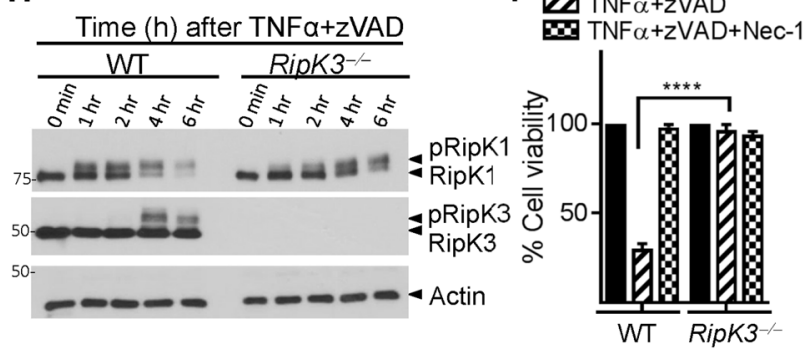

Fig. 2 TRIF and RipK3 promotes the degradation of necrosomal proteins. a WT and RipK $1^{K 45 A}$ mutant macrophages were treated with LPS + zVAD. Expression of various proteins was evaluated by western blotting of cell extracts at different time intervals post-stimulation. $\mathbf{b}$ Cell viability of WT and RipK $1{ }^{\mathrm{K} 45 \mathrm{~A}}$ macrophages was evaluated by MTT assay at $24 \mathrm{~h}$ post stimulation. $\mathbf{c}, \mathbf{d}$ WT, $\mathrm{TRIF}^{-\prime-}$, and MyD88 ${ }^{-\prime-}$ macrophages were treated with LPS + zVAD and at various time intervals cell extracts were collected and examined by western blotting (c). Cell viability was measured by MTT assay at $24 \mathrm{~h}$ post stimulation of cells with LPS $+\mathrm{zVAD}(\mathbf{d})$. e- $\mathbf{g}$ WT, RipK3 ${ }^{-/-}$, and $\mathrm{MLKL}^{-1-}$ macrophages were stimulated with LPS $+\mathrm{zVAD}$ and extracts were collected at various time intervals and tested for the expression of various proteins indicated in the figure (e, $\mathbf{g})$. Cell viability was measured by MTT assay at $24 \mathrm{~h}$ post stimulation of cells with LPS + ZVAD (f). $\mathbf{h}, \mathbf{i}$ WT and RipK3 ${ }^{-1-}$ macrophages were stimulated with TNFa + zVAD and western blot analysis was performed on cell extracts at various time intervals (h). Cell viability was evaluated by MTT assay at $24 \mathrm{~h}$ post stimulation (i). Graphs show the percentage of viable cells \pm SEM relative to controls. Each experiment was performed in triplicate and repeated three times. ${ }^{* *} P<0.001,{ }^{* * *} P<0.0001$

decrease in RipK1 phosphorylation by p38MAPK inhibition, this did not affect RipK1 degradation, and resulted in only partial reduction in RipK3 phosphorylation (Fig. S3 C, D), which did not impact necroptosis (Fig. S3 E). Inhibition of p38MAPK significantly reduced TNF $\alpha$ production (Fig S3 F). These results indicate that the phosphorylation of RipK1 itself is not required for the degradation of RipK1.

RipK3-inhibitor GSK843 ${ }^{36}$ that completely blocked the phosphorylation of RipK3 (Fig. 3b) and necroptosis (Fig. 3c-g), did not prevent the loss of RipK1 or Casp-8 (Fig. 3b). RipK1 inhibitor (Nec-1) and RipK3 inhibitor
(GSK872) showed similar inhibition of necroptosis as measured by MTT (Fig. 3c), CCK8 (Fig. 3d) assay, Zombie yellow staining for dead cells (Fig. 3e, f) and PI/Hoechst staining (Fig. 3g). Interestingly, GSK843 partially restored the levels of cIAP1/2 (Fig. 3b), similar to what was observed with MLKL-deficient cells (Fig. 2g). Similar results were observed with another RipK3 inhibitor (GSK872), which resulted in rescue of cIAP1/2, but not Casp-8 or RipK1 levels (Fig. S3 G, H).

Nec-1, when added at $3 \mathrm{~h}$ post-LPS/zVAD treatment was unable to block RipK1 degradation or death (Fig. 3h, i). We performed co-immunoprecipitation with an anti- 

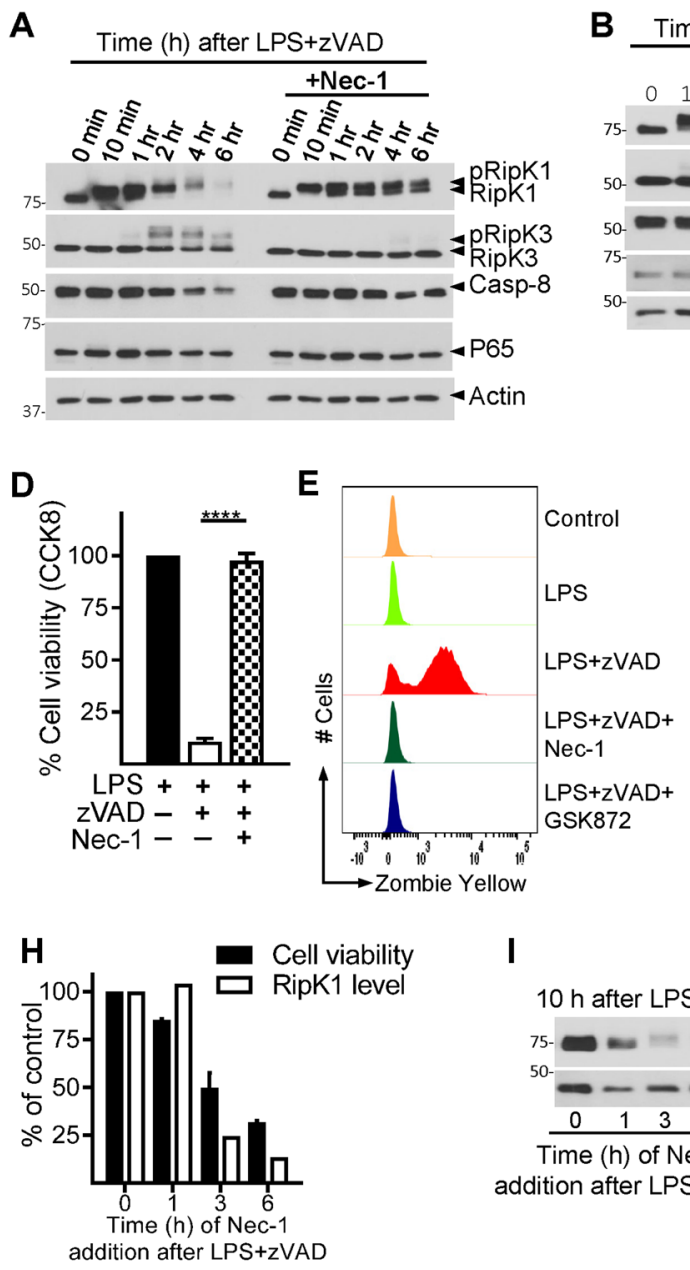
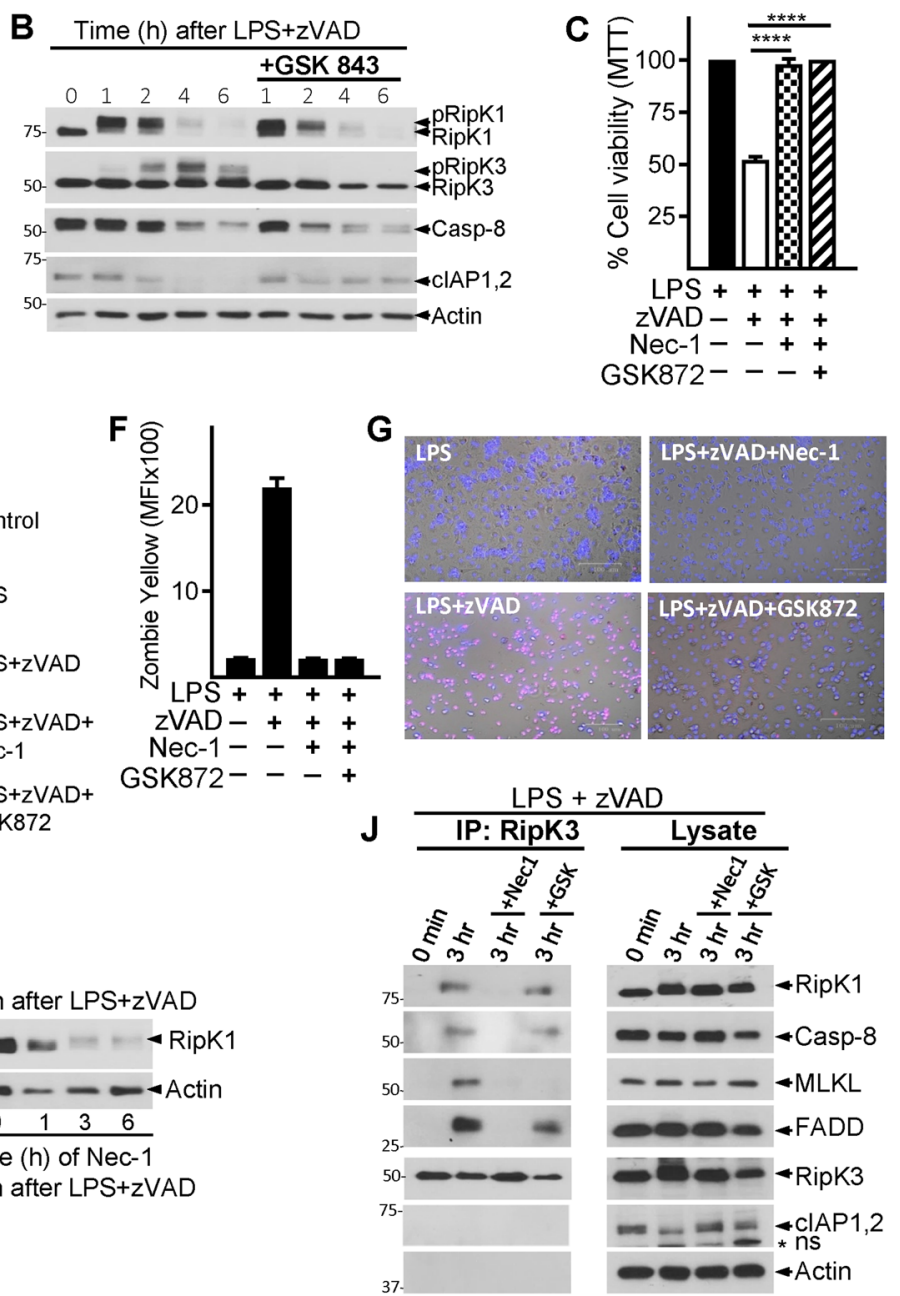

Fig. 3 RipK1-RipK3 interaction promotes the degradation of RipK1. a-d WT macrophages were stimulated with LPS + ZVAD in the presence or absence of Nec-1 (30 $\mu \mathrm{M})(\mathbf{a}, \mathbf{c}, \mathbf{d})$ or GSK843/872 $(3 \mu \mathrm{M})(\mathbf{b})$. Cell lysates were collected at varying time intervals and examined by western blotting $(\mathbf{a}$, b). Cell viability of macrophages was examined by MTT (c), CCK8 (d) assay, Zombie yellow staining (e, $\mathbf{f}$ ), and at $24 \mathrm{~h}$ post stimulation, or by staining cells with PI/Hoechst $(\mathbf{g})$ at $6 \mathrm{~h}$ post stimulation. $\mathbf{h}$, i Macrophages were treated with LPS + zVAD. Nec-1 was added immediately, or at various time intervals post stimulation with LPS + ZVAD. Cell viability (h) was measured at $24 \mathrm{~h}$ by MTT assay, and expression of RipK1 (i) was measured by western blotting of extracts collected at $10 \mathrm{~h}$ post stimulation with LPS + ZVAD. $\mathbf{j}$ RipK3 was immune-precipitated in control cells and in cells stimulated with LPS + zVAD for $3 \mathrm{~h}$ in the absence or presence of Nec-1 or GSK872. Expression of various proteins in the immune-precipitates and cell lysates were examined by western blotting. Graphs show the percentage of viable cells \pm SEM relative to controls. Each experiment was performed in triplicate and repeated three times. ${ }^{* * *} P<0.0001$

RipK3 antibody and evaluated the impact of inhibition by Nec-1 vs. GSK872 (Fig. 3j). Addition of Nec-1 completely blocked the association of RipK1, Casp-8, FADD, and MLKL with RipK3 in the necrosome. In contrast, addition of the RipK3-specific inhibitor (GSK872) blocked only the RipK3-MLKL interaction (Fig. 3j). Thus, RipK1-RipK3, but not RipK3-MLKL interaction is necessary for necrosomal degradation. Overall the results suggest the existence of two stages of necrosome signaling, an early necrosome consisting of RipK1, RipK3, Casp-8, FADD (and possibly other components), and a late necrosome complex that includes MLKL and is dependent on RipK3kinase activity.

\section{Degradation of necrosome impairs cell death and cytokine expression}

We treated cells with LPS + zVAD to induce necrosome signaling, but blocked cell death using Nec-1 or GSK872. Since Nec-1 and GSK872 have opposite impact on necrosome degradation (Fig. 3a, b), we could specifically disentangle the impact of necrosome degradation on susceptibility to necroptosis and impact on cytokine 

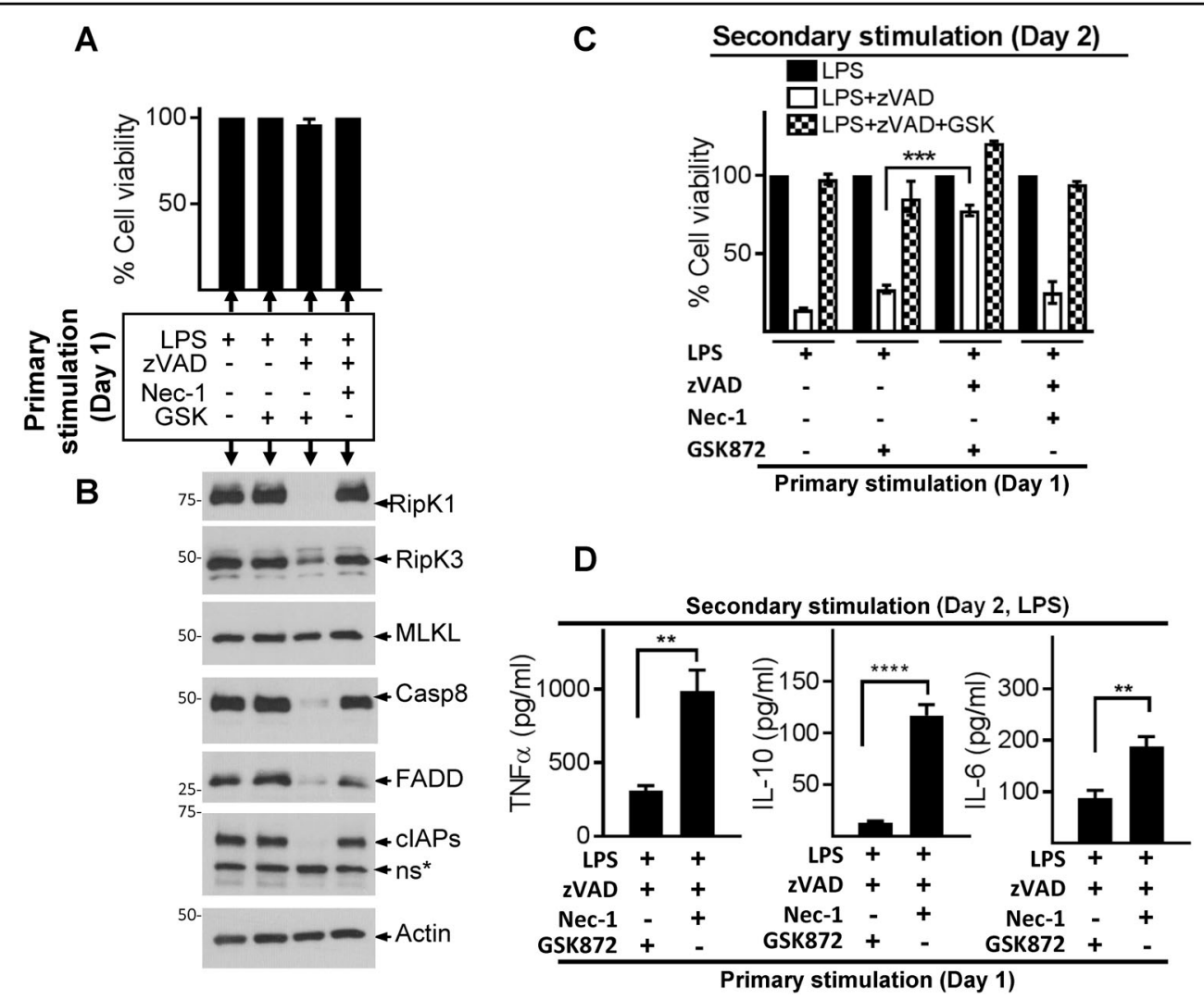

Fig. 4 Necrosome degradation results in resistance to cell death and impaired cytokine expression. a-d WT macrophages were stimulated as described in panel $\mathbf{a}$. Cell death was evaluated by MTT at $24 \mathrm{~h}$ post stimulation (a), and western blotting was performed in cell extracts collected at $24 \mathrm{~h}(\mathbf{b})$. After primary stimulation $(24 \mathrm{~h}$ ), cells were washed and stimulated with LPS, LPS + ZVAD, or LPS + zVAD + GSK872, and cell death was evaluated after another $24 \mathrm{~h}$ by MTT assay (c). Supernatants were collected at $6 \mathrm{~h}$ post secondary stimulation with LPS and expression of cytokines was measured by ELISA (d). Graphs show the percentage of viable cells \pm SEM relative to cells treated with LPS in the absence of zVAD. Each experiment was repeated thrice with triplicate samples. ${ }^{*} P<0.05,{ }^{* *} P<0.01,{ }^{* * *} P<0.001,{ }^{* * *} P<0.0001$

expression. Primary stimulation of macrophages for $24 \mathrm{~h}$ with LPS $+\mathrm{zVAD}$ and Nec-1 or GSK resulted in resistance to cell death as expected (Fig. 4a), although only treatment with RipK3 inhibitor (GSK872) resulted in a sustained loss of RipK1, Casp-8, cIAP1/2, and FADD expression (Fig. 4a, b).

We were intrigued to test the properties of macrophages with depleted levels of cell death mediators. Cells that expressed poor levels of necrosomal proteins due to previous treatment with LPS + zVAD and RipK3 inhibitor (GSK872) displayed potent resistance to induction of necroptosis (Fig. 4c). In contrast, cells previously treated with LPS, LPS + GSK872, or LPS + zVAD + Nec-1, which expressed high levels of necrosomal proteins were highly susceptible to necroptotic cell death (Fig. 4c). Degradation of necrosome during primary stimulation (LPS + zVAD + GSK872) also resulted in significantly reduced expression of cytokines in response to secondary stimulation (Fig. 4d). These results reveal that early degradation of the necrosome is a mechanism of cellular escape from necroptosis and cytokine expression.

\section{RipK3 interaction promotes K48-ubiquitination of necrosome components}

We tested whether a proteasome inhibitor, Lactacystin, could rescue the loss of the RipK1/Casp- 8 levels. Indeed, Lactacystin induced substantial rescue of RipK1 levels (Fig. 5a, b), which corresponded to an increase in the phosphorylation of RipK3 (Fig. 5a, b). Caspase- 8 and cIAP1/2 were partially rescued by Lactacystin treatment (Fig. 5a, b). Similar results were obtained with another proteasome inhibitor, MG341 (Fig. S4 A). Inhibition of transcription (Actinomycin D), endosomal acidification (Chloroquine) and autophagy (hydroxychloroquine) did not rescue the levels of RipK1 (Fig. S4 B).

We immunoprecipitated ubiquitinated proteins with "tandem ubiquitination binding entities" (TUBEs) and performed western blotting of immune-precipitates. We 


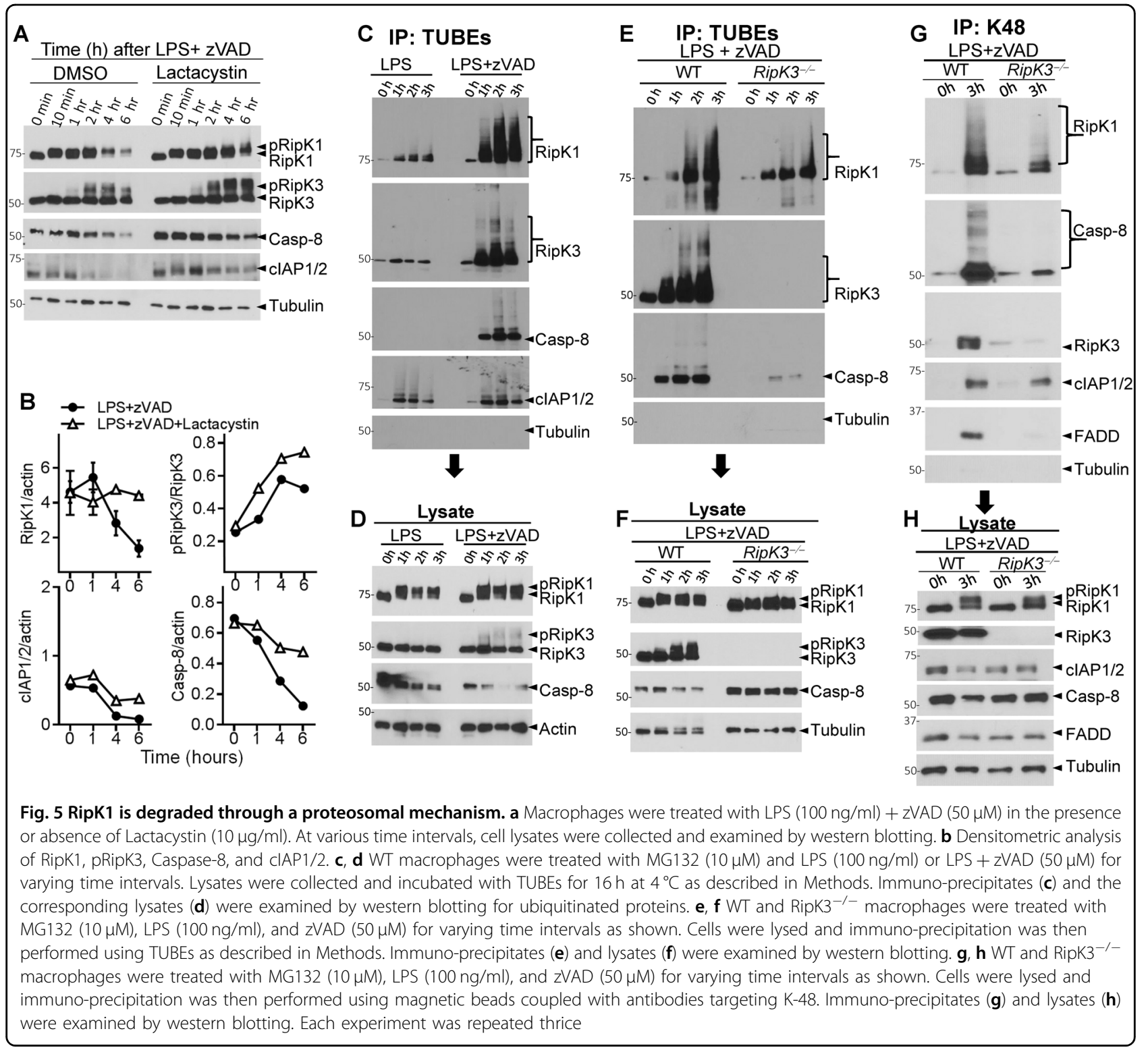

observed that RipK1 was profoundly ubiquitinated following stimulation by LPS + zVAD, but not by LPS (Fig. 5c, d). Similar results were observed when cells were stimulated with TNF $\alpha+$ zVAD (Fig. S4 C, D). Ubiquitination of RipK1 following LPS + zVAD stimulation was significantly reduced in RipK3-deficient macrophages (Fig. 5e, f). Using K48-ubiquitin specific antibody to immuno-precipitate proteins, we observed extensive K48 ubiquitination of RipK1 and Caspase- 8 following necrosome activation, which was highly reduced in RipK3deficient macrophages (Fig. 5g, h). These results show that association of RipK1 and RipK3 in the early necrosome leads to ubiquitination of necrosome components and drives degradation of interacting proteins.
IAPs do not promote the maintenance of RipK1 during necrosome signaling

Since cIAPs promote K63-ubiquitination of RipK $1^{37}$, we evaluated the role of cIAPs in necrosome degradation. The loss of RipK1 during necrosome signaling remained unperturbed in cIAP1- or cIAP2-deficient macrophages (Fig. 6a, b). Necroptosis of macrophages was also not significantly modulated in cIAP1/2-deficient cells (Fig. 6c). Similarly, the loss of RipK1 or necroptosis was also not influenced by the deficiency of XIAP (Fig. 6d, e). We also pre-treated macrophages for $1 \mathrm{~h}$ with the SMAC mimetic Birinapant (BP) which induces rapid degradation of both cIAPs $^{38,39}$. Treatment of macrophages with BP followed by LPS $+\mathrm{zVAD}$ resulted in loss of both cIAPs 


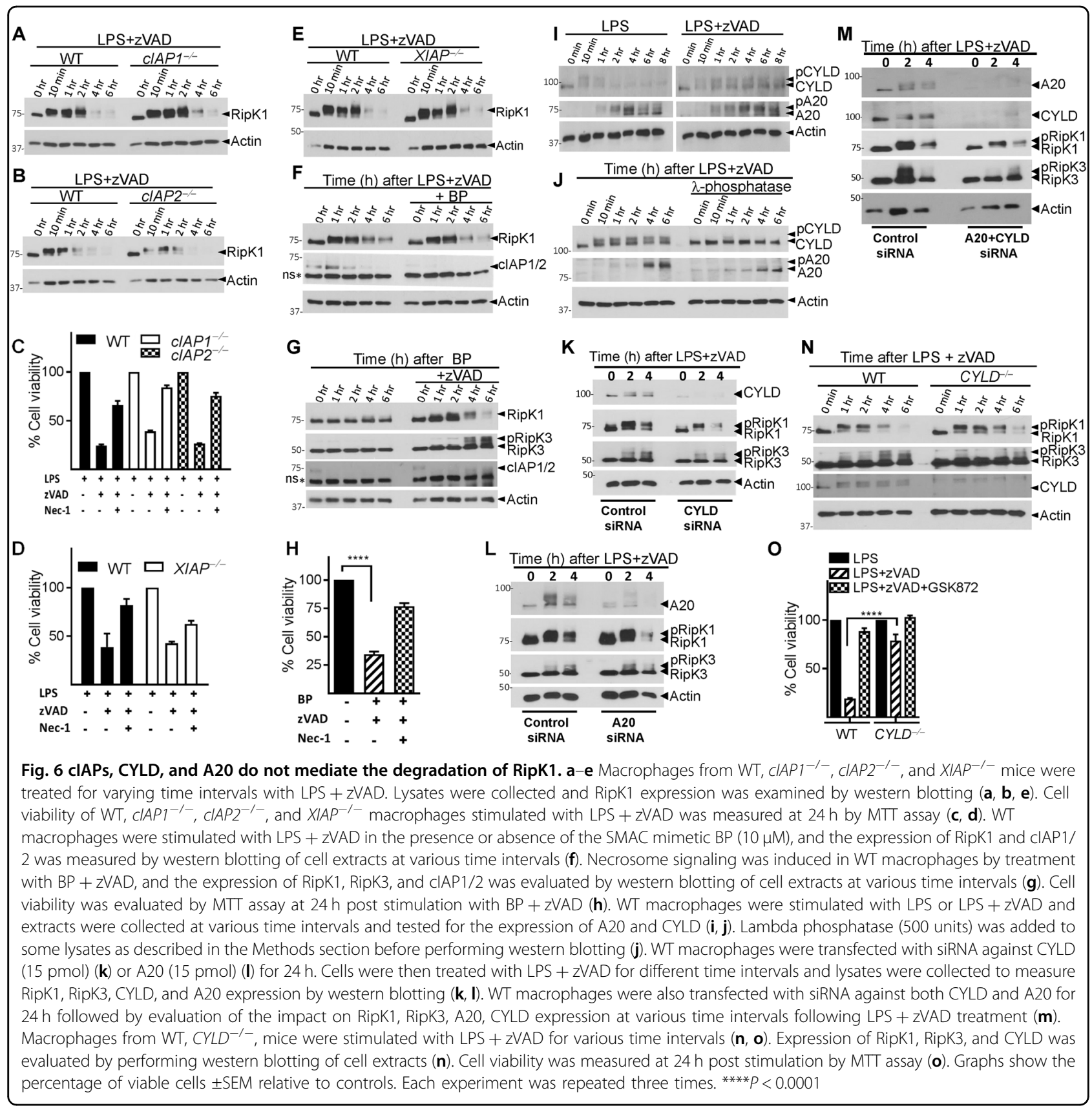

but did not affect the degradation of RipK1 (Fig. 6f). Induction of necroptosis by $\mathrm{BP}+\mathrm{zVAD}$ treatment also resulted in degradation of RipK1 (Fig. 6g, h).

\section{A20 and CYLD do not impact the degradation of RipK1 during necrosome signaling}

In fibroblasts CYLD and A20 have been shown to edit the ubiquitination of RipK $1^{40,41}$. We observed that the expression of CYLD was increased during necrosome signaling (Figs. 1k and 6i, and Fig. S5 A). CYLD and A20 were phosphorylated during necrosome signaling (Fig. 6j). Phosphorylation of CYLD has been reported to inhibit its deubiquitinase (DUB) activity ${ }^{42-44}$. We performed CYLD and A20 knock-down separately or in combination and evaluated the impact on RipK1 degradation. Rather than inhibiting RipK1 degradation, knock-down of CYLD and/ or A20 appeared to enhance the degradation of RipK1 (Fig. 6k-m and Fig. S5 C, D), suggesting that CYLD and A20 promote maintenance of RipK1. Similarly, CYLDdeficient macrophages had little change in degradation of RipK1, although phosphorylation of RipK3 and necroptosis was significantly lower relative to WT macrophages (Fig. 6n, o). Inhibition of the linear ubiquitin chain assembly complex (LUBAC) by gliotoxin ${ }^{45}$ also did not 

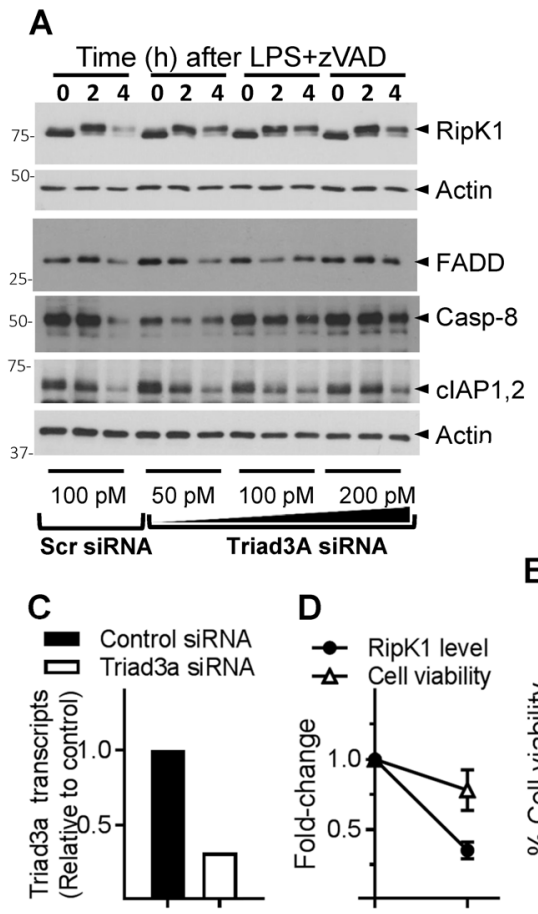

D

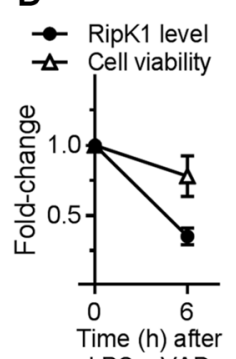

LPS+ZVAD
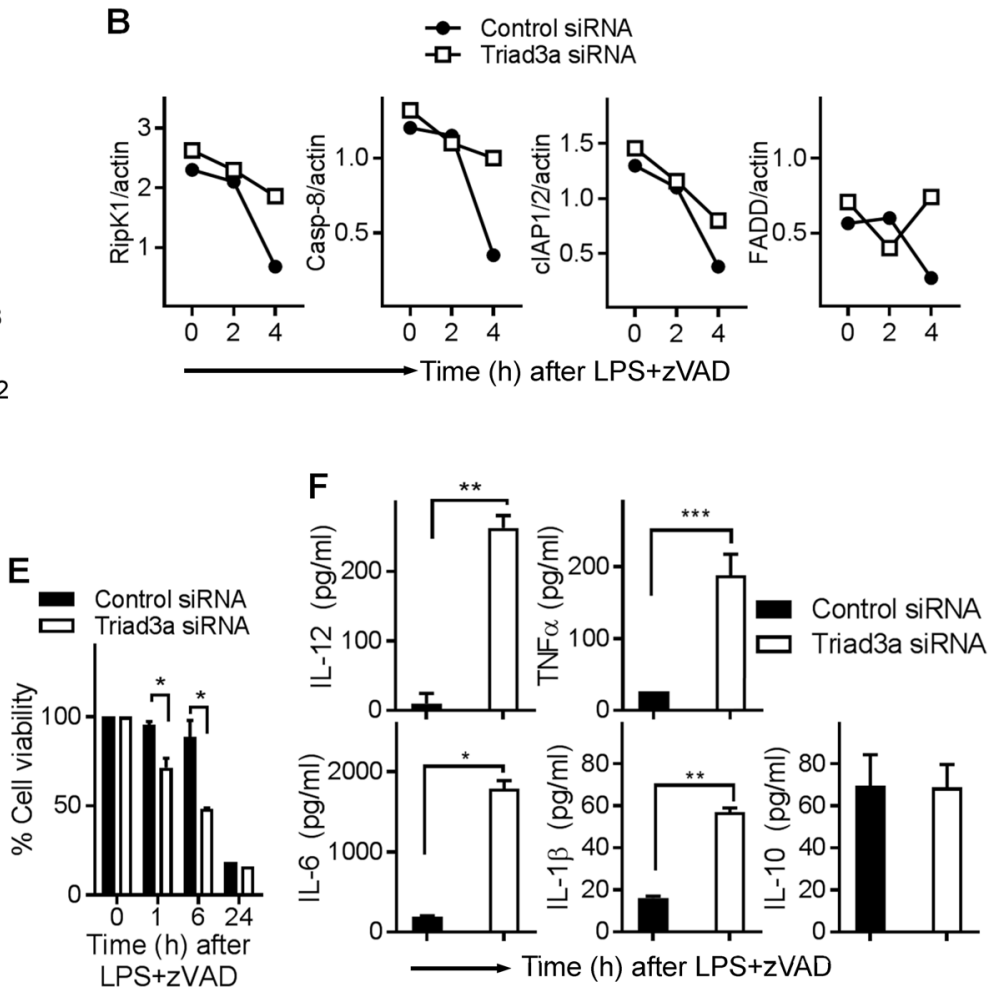

Fig. 7 Triad3a promotes the degradation of necrosome and regulates necroptosis and cytokine expression. a-e WT macrophages were transfected with siRNA against Triad3a (50, 100, or 200 pmol) for $24 \mathrm{~h}$. Control cells were transfected with scrambled siRNA (100 pmol). Cells were then treated with LPS + ZVAD for different time intervals and lysates collected for western blotting (a). Densitometric analysis of representative western blots transfected with $100 \mathrm{pM}$ siRNA was performed (b). Expression of Triad3a was evaluated at $24 \mathrm{~h}$ post transfection by qRT-PCR (c). WT macrophges were treated with LPS $(1 \mathrm{ng} / \mathrm{ml})+\mathrm{ZVAD}(50 \mu \mathrm{M})$, and the impact on cell death (MTT assay) and RipK1-expression (western blotting) evaluated (d). Primary macrophages were transfected with control or Triad3a siRNA for $24 \mathrm{~h}$, and cells were washed next day and cell death was evaluated at various time intervals post stimulation of cells with LPS $(1 \mathrm{ng} / \mathrm{ml})+\mathrm{zVAD}(50 \mu \mathrm{M})$ by MTT assay $(\mathbf{e})$. Macrophages were transfected with siRNA against Triad3a as mentioned above. At $24 \mathrm{~h}$, cells were washed and stimulated with LPS $(100 \mathrm{ng} / \mathrm{ml})+\mathrm{zVAD}(50 \mu \mathrm{M})$. Cytokines were measured in cell supernatants by ELISA (f). Graphs show the percentage of viable cells \pm SEM relative to cells treated with LPS in the absence of $z V A D$. Each experiment was repeated thrice with triplicate samples. ${ }^{*} P<0.05,{ }^{* *} P<0.01$

have any impact on the degradation of RipK1 or necroptosis (Fig. S5 F, G).

\section{Triad3a promotes necrosome degradation and regulates cell death and cytokine production}

Stimulation of cells with LPS/zVAD initiated cell death without any evidence of RipK1 degradation for the first few hours (Fig. S6 A, B). There was a deceleration in the magnitude of cell death between 3 and $8 \mathrm{~h}$ that correlated with a precipitous decrease in necrosomal proteins, suggesting that the loss of necrosomal proteins may act as a regulatory mechanism to limit cell death. Triad3a is an E3 ubiquitin ligase that interacts with TLRs ${ }^{46}$, TRIF/RipK $1^{47}$ and regulates TLR signaling ${ }^{46}$. Since necrosome signaling and degradation was inhibited in $T^{2} I F^{-1}$ macrophages (Fig. 2c, d), we evaluated whether Triad3a impacts the degradation of RipK1 during necrosome signaling. Knockdown of Triad3a by siRNA resulted in reduction in the degradation of RipK1, FADD, and Caspase-8 (Fig. 7a-c), indicating that Triad3a promotes the degradation of these proteins during necrosome signaling. Knock-down of Triad3a did not have a significant impact on the degradation of cIAP1/2 (Fig. 7a, b). In the absence of any highquality western blot antibody that could specifically detect mouse Triad3a, we tested the efficiency of Triad3a knockdown by qRT-PCR, which showed substantial reduction in Triad3a levels (Fig. 7c). Stimulation of cells with LPS + zVAD had a marginal impact on cell death at earlier time periods (Fig. 7d, e), knock-down of Triad3a resulted in slightly enhanced cell death (Fig. 7e). Triad3a knockdown resulted in substantial increase in the expression of inflammatory cytokines following necrosome signaling (Fig. 7f). Overall, these results indicate a model wherein recruitment of RipK3 to the early necrosome leads to Triad3a-dependent K48 ubiquitination of RipK1 and other necosome components as an auto-regulatory mechanism that limits both cell death and inflammatory cytokine production. 


\section{Discussion}

Since macrophages promote inflammatory responses in various tissues, and necrosome signaling leads to exacerbation of inflammatory responses ${ }^{13,48,49}$, understanding the mechanisms of regulation of necrosome signaling in macrophages is crucial. The mechanisms through which RipK1 and RipK3 are phosphorylated during necrosome signaling are unclear ${ }^{14,50}$. TLR signaling via TRIF has been shown to promote phosphorylation of RipK1 in macrophages $^{51}$, however, our results indicate that TRIFdeficient macrophages had no impairment in phosphorylation of RipK1. Inhibition of RipK1 by Nec- $1^{50}$ also did not abolish the phosphorylation of RipK1. Furthermore, RipK1 ${ }^{\mathrm{K} 45 \mathrm{~A}}$ kinase-inactive macrophages also had no impairment in phosphorylation of RipK1, supporting a view that RipK1 does not auto-phosphorylate itself. IKK complex has been previously shown to phosphorylate RipK $1^{52}$. MK2, the downstream target of p38-MAPK was shown to mediate an inhibitory phosphorylation on RipK $1{ }^{53}$. These results support a complex model of RipK1 phosphorylation, wherein multiple kinases can phosphorylate RipK1 at multiple sites which can both inhibit and activate necroptotic cell death ${ }^{52,54}$.

Downstream of RipK1, we and others have previously shown that TRIF signaling is necessary for phosphorylation of RipK3 in macrophages ${ }^{30,51}$. Interestingly, here we observe that Nec-1 inhibited the interaction of RipK1 and RipK3, and blocked the phosphorylation of RipK3, leading to inhibition of necroptosis and rescue of degradation of various necrosomal proteins. While previous results have been interpreted to indicate that RipK1 can phosphorylate RipK3, our results support a view that Nec-1 blocks RipK1-RipK3 interaction and does not necessarily impact on kinase function. More recently, convincing evidence has emerged to show that RipK3 dimerization leads to auto-phosphorylation, which is key for downstream activation of MLKL ${ }^{55,56}$. We add here a curious observation that the phosphorylation of RipK3 is abrogated in MLKLdeficient macrophages, suggesting that RipK3-MLKL platform may facilitate RipK1-mediated phosphorylation or auto-phosphorylation of RipK3.

Our results indicate that while the kinase function of RipK3 is necessary to activate necroptosis, interaction with RipK3 leads to the degradation of RipK1 and other necrosomal proteins. This represents a complex model wherein RipK3 appears to have contrasting functions. Our results suggest that the formation of an early necrosome platform that initiates a decision between degradation via K48-ubiquitination or cell death via RipK3 kinase activity and MLKL recruitment.

Several ubiquitin ligases have been shown to ubiquitinate RipK1 in TNF-R complex-I, including cIAPs, TRAF2, and LUBAC. All of these ubiquitinations in the complex I (M1- or K63-ubiquitin) appear to promote
RipK1 signaling, not degradation ${ }^{57}$. Consistent with this, our results indicate that treatment of cells with TNF $\alpha$ or LPS alone does not induce RipK1 degradation. Rather, we find that RipK1 degradation only occurs following association with RipK3, and is impacted by Triad3a. Interestingly, Triad3a has been shown to cause degradation and regulation of TLR signaling, including RipK $1^{46,47}$. Due to the lack of availability of a suitable antibody, we have been unable to confirm whether RipK3 directly recruits Triad3a to the necrosome complex.

Various DUBs such as A20 and CYLD remove the ubiquitination chains on RipK1 as the cells transition toward the necrosome signaling platform ${ }^{58,59}$. Our results show that the expression and phosphorylation of CYLD increased during necrosome signaling. Interestingly, phosphorylation of CYLD has been shown to inhibit its DUB activity ${ }^{42-44}$.

Casp- $8^{60,61}$, cIAPs $^{38}$, and Cathepsins ${ }^{62}$ regulate necroptosis in macrophages. Here we have introduced a new mechanism of self-regulation built into necroptotic signaling. Similar to the inactivating timer mechanism identified in the apoptosome ${ }^{63}$, we reveal here that recruitment of RipK3 to the necrosome initiates a competition between necrosomal degradation and necrosomal maturation. This would suggest that the rate of RipK3 kinase activation and MLKL recruitment determine the proportion of necroptotic death that occurs. By applying small molecule inhibitors of RipK3, we were able to prevent activation of the late necrosome, resulting in cells with degraded necrosomal machinery and resistance to subsequent necroptotic challenge.

RipK $1^{19,33}$ and RipK $3^{13,17,30}$ promote inflammatory responses, and it is not clear whether this is related to the role of these proteins outside the necrosome complex ${ }^{64,65}$. In contrast to apoptosis, which is considered to be immunologically silent or immune-suppressive ${ }^{66}$, the disruption of the cellular membrane and release of DAMPs during necroptosis is expected to promote inflammatory responses ${ }^{67}$. Our data indicate that proteasomal degradation of RipK1 and other early necrosome components acts to destabilize death inducing complexes and prevent the completion of the necroptosis program. It is conceivable that this could result in a pool of cells that survive cell death induction and maintain key functionality.

\section{Methods \\ Mice}

C57BL6/J (Jax \#000664), TRIF ${ }^{-/}$(Jax \#005037), Myd88 ${ }^{-/-}$(Jax \#009088), and TNFR1/2 ${ }^{-/-}$(Jax \#003243) were obtained from Jackson Labs (Bar Harbor, USA). RipK3 ${ }^{-/-}$were a kind gift of Dr. Vishva Dixit (Genentech, San Francisco, CA, USA); RipK $1^{\mathrm{K} 45 \mathrm{~A}}$ mice and wild type litter mates were a kind gift of Dr. Peter J. Gough 
(GlaxoSmithKline, Collegeville, USA); $M L K L^{-/-}$bone marrow samples were received from Dr. Emad Alnemri (Thomas Jefferson University, Philadelphia, USA). $C Y L D^{-1-}$ mice were as previously described ${ }^{68}$. Experiments were performed in accordance with the Canadian Council on Animal Care guidelines and the Ethics Board and/or the Animal Care Committee at University of Ottawa.

\section{Reagents}

Pan-caspase inhibitor, z-VAD-fmk (\#A1902) was obtained from ApexBio (Houston, USA). Lactacystin (L6785), MG132 (C2211), cyclohexamide (C4859), hydroxychloroquine (H0915), and ultrapure LPS (E. coli 0111:B4, L3024) were obtained from Sigma-Aldrich (Oakville, Canada). Necroatatin-1 (9037) was obtained from Sigma Chem Co. (Oakville, Canada). M-CSF (416-ML) and TNF $\alpha$ (410-MT) were obtained from R\&D (Minneapolis, USA). Birinapant (S7015) was obtained from Selleckchem (Houston, USA). GSK843 and GSK'872 (AOB4898, AOB488) were obtained from Aobious (Gloucester, USA). Caspase-8 Inhibitor, z-IETD-FMK (064-20C) was obtained from BioVision (San Francisco, USA). P38 inhibitor (5633S) was obtained from Cell Signaling (Danvers, USA). Alkaline Phosphatase, Calf Intestinal (CIP) (M0290, NEB). Protease inhibitors (04693132001) were obtained from Roche Applied Science (Laval, Canada). PR-619 (SI9619), 1,10phenanthroline (SI9649) and GST-tagged tandem ubiquitin-binding entities (TUBE) (UM102) were obtained from LifeSensors (Malvern, USA). Actinomycin-D (0219452505) was obtained from MP Biomedicals (Solon, USA). Necrostatin-1s (cat\# 504297) and MG341 (Bortezomib) (cat\# 504314) were obtained from Calbiochem (San Diego, CA, USA). Zombie Yellow ${ }^{\text {rn }}$ Fixable Viability Kit cat\# 423103, Biolegend (San Diego, USA).

\section{Generation of macrophages}

Primary bone marrow-derived macrophages (BMDMs) were differentiated in the presence of $5 \mathrm{ng} / \mathrm{ml}$ of M-CSF for 6 days at $37^{\circ} \mathrm{C}$ in R8 media (RPMI, $8 \%$ fetal bovine serum, $\quad 50 \mu \mathrm{M} \quad \beta$-mercaptoethanol and $50 \mathrm{ng} / \mathrm{ml}$ gentamycin) ${ }^{30}$.

\section{Cell culture and viability assay}

BMDMs were stimulated in either 24- or 96-well tissue culture plates with LPS $(100 \mathrm{ng} / \mathrm{ml})$ or TNF- $\alpha$ (1000 U/ $\mathrm{ml})$, in the presence of pan-caspase inhibitor zVAD (50 $\mu \mathrm{M})$. In some experiments, BMDM were co-treated with various inhibitors and agonists $30 \mathrm{~min}$ before stimulation and left for a couple of hours before assaying the cell viability or collecting protein lysate. Cell viability was measured using 3-[4,5-dimethylthiazol-2-yl]-2,5-diphenyltetrazolium bromide (MTT) assay. The MTT reagent was diluted with R8 media at a final concentration $0.5 \mathrm{mg} /$ $\mathrm{ml}$ and incubated at $37^{\circ} \mathrm{C}$. After $2 \mathrm{~h}, 5 \mathrm{mM} \mathrm{HCl}$ isopropyl alcohol were added to solubilize MTT crystals and absorbance was measured at a wavelength of $570 \mathrm{~nm}$ with a reference wavelength of $650 \mathrm{~nm}$ on a Molecular Devices FilterMax plate reader. Cell Counting Kit-8 (CCK-8) was used and $10 \mu \mathrm{l}$ was added to each well and incubated at $37^{\circ} \mathrm{C}$ for $2-4 \mathrm{~h}$. After incubation the absorbance was measured at $450 \mathrm{~nm}$ using Molecular Devices FilterMax plate reader. CCK-8 (cat\# CK04-01) was obtained from Dojindo Molecular Technologies (Rockville, USA).

\section{Flow cytometry}

Zombie Yellow $^{\text {tix }}$ (BioLegend, San Diego, CA) was used to assess cell viability. Using BPS buffer, $24 \mathrm{~h}$ after treating the cells in a 96-well plate with various treatments, the seeded cells were washed twice. Without detaching the cells, $50 \mu \mathrm{l}$ of diluted Zombie Yellow ${ }^{\mathrm{ma}}$ solution (1:100 in PBS) was added into each well and the plates were incubated at room temperature, in the dark, for 15-30 min before washing with $100 \mu$ FACS buffer (PBS, $1 \%$ BSA, 1 mM EDTA) once. Then, samples were fixed by using $1 \%$ paraformaldehyde and analyzed with a LSR Fortessa cell analyzer and FACSDiva software (Becton Dickinson). The data were analyzed with FlowJo software.

\section{Quantitative RT-PCR}

Total RNA was isolated using TRIzol reagent (Life Technologies). Isolated RNA was reverse transcribed into cDNA in a $20-\mu \mathrm{l}$ reaction volume using SuperScript III Reverse Transcriptase (Invitrogen,18080- 044) as follows: $1 \mu \mathrm{g}$ of RNA template was added to $0.5 \mu \mathrm{l}$ of oligo(dT) (50 $\mu \mathrm{M}), 0.5 \mu \mathrm{l}$ of random primers $(50-250 \mathrm{ng}), 4 \mu \mathrm{l}$ of $5 \times$ First Strand buffer, $1 \mu \mathrm{l}$ of DTT $(0.1 \mathrm{M}), 1 \mu \mathrm{l}$ of dNTP (10 $\mathrm{mM}), 1 \mu \mathrm{l}$ of RNase OUT (40 units/ $\mu \mathrm{l}$ ), and $1 \mu \mathrm{l}$ of SuperScript III (200 units/ $\mu \mathrm{l})$. After cDNA synthesis, $2 \mu \mathrm{l}$ of cDNA was analyzed using the SYBR Green (Life Technologies) fast method performed on an Applied Biosystems 7500 quantitative RT-PCR system. The primers used were as follows: Actin (forward) $5^{\prime}$-GAT CAAGATCATTGCTCCTCCTG-3', (reverse) 5'-AGGG TGTAAAACGCAGCTCA-3'; RipK1 (forward) 5'-GGCC AACATTTCTTGGCATTGA-3', (reverse) 5'-CTGCAG CACTGGGCTTTGAT-3'; Caspase-8 (forward) 5'-TGCC CAGATTTCTCCCTACA-3', (reverse) 5' -AAGCAGGCT CAAGTCATCTTCC-3'. Triad3a (forward) 5'-ACAGA TGATCACCATGTTTGGTT-3', (reverse) 5'-CATCCA TTCCTTTCCCCGGT-3'.

\section{RNA interference}

The smart pool ON-TARGETplus siRNA against CYLD, A20, and TRIAD3A were purchased from Dharmacon (GE Healthcare, USA). BMDMs were transfected with siRNA using Lipofectamine ${ }^{\circledast}$ RNAiMAX Transfection Reagent (13778030, Invitrogen) according to the 
manufacturer's instructions. After $24 \mathrm{~h}$, the transfected cells were used for time course experiments. Sequence of the siRNAs is provided in the supplemental section.

\section{Western blotting}

Cells were stimulated as indicated and lysed in SDS lysis buffer with $1 \% \beta$-mercaptoethanol and boiled immediately at $95^{\circ} \mathrm{C}$ for $5 \mathrm{~min}$. Lysates were separated by $10 \%$ or 8\% SDS-PAGE and transferred to PVDF membrane. Immunoblot analysis was performed using the following antibodies: mouse anti-RipK1 (610458, BD Biosciences, Mississauga, Canada), rabbit anti-RipK3 (2283, ProSci Inc., Poway, USA), mouse anti-actin (47778, Santa Cruz Biotechnology, Dallas, USA), rabbit anti-STAT1 (9172, Cell Signaling), rat anti-caspase-8 (1G12, Enzo), rabbit anti-CIAPs (CY-P1041, Cyclex), rat anti-MLKL monoclonal (MABC604, EMD Millipore), rabbit anti-CYLD (8462, Cell Signaling), rabbit anti-A20 (5630, Cell Signaling, Danvers, USA), mouse anti-FADD (1F7, Enzo, Farmingdale, USA) and mouse anti- $\alpha$ Tublin (5286, Santa Cruz Biotechnology, Dallas, USA). Rabbit anti-PP2A B subunit $(100 \mathrm{C} 1)$ was obtained from Cell Signaling Inc. In some cases, whole cell lysates were prepared in RIPAEDTA free buffer supplemented with protease inhibitor and 50 Units of calf intestinal phosphatase (CIP) was used to dephosphorylate the total protein in $1 \times$ CutSmart NEB buffer for $50 \mathrm{~min}$ at $37^{\circ} \mathrm{C}$. SDS lysis buffer was added to the treated samples and boiled for $5 \mathrm{~min}$ at $95^{\circ} \mathrm{C}$ followed by standard western blot protocol.

\section{Immunoprecipitations}

Cell lysates were immunoprecipitated with Dynabeads co-immunoprecipitation kit (Invitrogen, Life Technologies, Burlington, Canada) with the following antibodies: rabbit anti-RipK3 (2283, ProSci Inc., Poway, USA), rabbit anti-K48 ubiquitin (Apu2, Millipore, USA), and rabbit anti-K63 ubiquitin (Apu3, Millipore, Burlington, Canada). Bound proteins were eluted and subsequently analyzed by western blot standard protocol. The densitometric quantification of western blot signals was performed using Image 1.48 software (Maryland, USA).

For immune-precipitation with tandem ubiquitinbinding entities (TUBES), BMDMs were treated with proteasome inhibitor MG132 $(5 \mu \mathrm{g} / \mathrm{ml})$ before stimulation with LPS/zVAD or TNF/zVAD. Cells were washed with ice-cold $1 \times \mathrm{PB}$ and lysed in buffer contains $50 \mathrm{mM}$ TrisHCL pH 7.5, 150 mM NaCl, 1 mM EDTA, 1\% NP-40, 10\% glycerol, protease inhibitor, phosphatase inhibitor, $20 \mu \mathrm{M}$ deubiquitinase inhibitor PR-619 and 1× 1,10-phenanthroline. The lysates were sonicated for $10 \mathrm{~s} 3$ times and then centrifuged at $14,000 \times g$ for $30 \mathrm{~min}$ at $4{ }^{\circ} \mathrm{C}$ to pellet cell debris. The clarified lysate were then incubated with equilibrated Agarose-TUBEs for $16-24 \mathrm{~h}$ at $4{ }^{\circ} \mathrm{C}$ on a rocker platform. After incubation, the beads were collected by low speed centrifugation $5000 \times g$ for $5 \mathrm{~min}$ at $4{ }^{\circ} \mathrm{C}$. The unbound fractions were saved at $-20^{\circ} \mathrm{C}$ and the beads were washed 3 times with ice-cold TBS-T washing buffer that contains $20 \mathrm{mM}$ Tris-HCL pH 8, $150 \mathrm{mM}$ $\mathrm{NaCl}$ and $0.1 \%$ Tween-20. The resin was re-suspended in $40 \mu \mathrm{l}$ of $2 \times$ SDS lysis buffer and boiled for $5 \mathrm{~min}$ at $95^{\circ} \mathrm{C}$ then centrifuged at $13,000 \times g$ for $5 \mathrm{~min}$ at room temperature. The eluent was analyzed by standard western blot protocol.

\section{Cytokine analysis}

Supernatants were collected from 24-well plates and the expression of cytokines was assessed using the BD optEIA ELISA kit (eBioscience, CA, USA) according to the manufacturer's instructions. The absorbance was read at $450-570 \mathrm{~nm}$ on a FilterMax F5 Multimode microplate reader (Molecular Devices).

\section{Statistical analyses}

All graphs show the average result taken from at least three independent experiments. Error bars show the standard error of the mean, and statistical significance between groups were determined by using Student's $t$-test using Graphpad Prism 6.07 software package (La Jolla, California, USA).

\section{Acknowledgements}

This work was supported by an operating grant from the Natural Sciences and Engineering Research Council (NSERC) to S.S., a grant from the National Institutes of Health (NIH, \#AR055398) to E.A. N.A.A. was supported by College of Applied Medical Sciences, King Saud University. S.M. was supported by a post-doctoral fellowship from CIHR.

\section{Author details}

${ }^{1}$ Department of Biochemistry, Microbiology, and Immunology, Faculty of Medicine, University of Ottawa, Ottawa, Canada. ${ }^{2}$ Applied Medical science, King Saud University, Riyadh, Saudi Arabia. ${ }^{3}$ Human Health and Therapeutics, National Research Council of Canada, Ottawa, Canada. ${ }^{4}$ Children's Hospital of Eastern Ontario Research Institute, Ontario, Canada. ${ }^{5}$ Department of Immunology, The University of Texas MD Anderson Cancer Center, Houston, TX, USA. ${ }^{6}$ Thomas Jefferson University, Philadelphia, PA, USA. ${ }^{7}$ Centre for Infection, Immunity and Inflammation, University of Ottawa, Ontario, Canada

Conflict of interest

The authors declare that they have no conflict of interest.

\section{Publisher's note \\ Springer Nature remains neutral with regard to jurisdictional claims in published maps and institutional affiliations.}

Supplementary Information accompanies this paper at (https://doi.org/ 10.1038/s41419-018-0672-0).

Received: 9 February 2018 Revised: 24 April 2018 Accepted: 27 April 2018 Published online: 22 May 2018

\footnotetext{
References

1. Belosevic, M., Davis, C. E., Meltzer, M. S. \& Nacy, C. A. Regulation of activated macrophage antimicrobial activities. Identification of lymphokines that
} 
cooperate with IFN-gamma for induction of resistance to infection. J. Immunol. 141, 890-896 (1988).

2. Bellamy, R. The natural resistance-associated macrophage protein and susceptibility to intracellular pathogens. Microbes Infect. 1, 23-27 (1999).

3. Ginhoux, F. \& Jung, S. Monocytes and macrophages: developmental pathways and tissue homeostasis. Nat. Rev. Immunol. 14, 392-404 (2014).

4. Salcedo, S. P., Noursadeghi, M., Cohen, J. \& Holden, D. W. Intracellular replication of Salmonella typhimurium strains in specific subsets of splenic macrophages in vivo. Cell Microbiol. 3, 587-597 (2001).

5. Dougan, G., John, V., Palmer, S. \& Mastroeni, P. Immunity to salmonellosis. Immunol. Rev. 240, 196-210 (2011).

6. Arpaia, N. et al. TLR signaling is required for Salmonella typhimurium virulence. Cell 144, 675-688 (2011).

7. Akira, S. TLR signaling. Curr. Top. Microbiol. Immunol. 311, 1-16 (2006).

8. O'Neill, L. A. Toll-like receptor signal transduction and the tailoring of innate immunity: a role for Mal? Trends Immunol. 23, 296-300 (2002).

9. Eckmann, L. \& Kagnoff, M. F. Cytokines in host defense against Salmonella. Microbes Infect. 3, 1191-1200 (2001).

10. Green, D. R. Apoptotic pathways: ten minutes to dead. Cell 121, 671-674 (2005).

11. Bidere, N., Su, H. C. \& Lenardo, M. J. Genetic disorders of programmed cell death in the immune system. Annu. Rev. Immunol. 24, 321-352 (2006).

12. Brennan, M. A. \& Cookson, B. T. Salmonella induces macrophage death by caspase-1-dependent necrosis. Mol. Microbiol. 38, 31-40 (2000).

13. Duprez, L. et al. RIP kinase-dependent necrosis drives lethal systemic inflammatory response syndrome. Immunity 35, 908-918 (2011).

14. Declercq, W., Vanden Berghe, T. \& Vandenabeele, P. RIP kinases at the crossroads of cell death and survival. Cell 138, 229-232 (2009).

15. Monack, D. M. et al. Salmonella exploits caspase-1 to colonize Peyer's patches in a murine typhoid model. J. Exp. Med. 192, 249-258 (2000).

16. Patel, R. et al. Culling of APCs by inflammatory cell death pathways restricts TIM3 and PD-1 expression and promotes the survival of primed CD8 T cells. Cell Death Differ. 24, 1900-1911 (2017).

17. Robinson, N. et al. Type I interferon induces necroptosis in macrophages during infection with Salmonella enterica serovar Typhimurium. Nat. Immunol. 13, 954-962 (2012).

18. Upton, J. W., Kaiser, W. J. \& Mocarski, E. S. Virus inhibition of RIP3-dependent necrosis. Cell Host Microbe 7, 302-313 (2010).

19. Shutinoski, B. et al. K45A mutation of RIPK1 results in poor necroptosis and cytokine signaling in macrophages, which impacts inflammatory responses in vivo. Cell Death Differ. 23, 1628-1637 (2016).

20. Cho, Y. S. et al. Phosphorylation-driven assembly of the RIP1-RIP3 complex regulates programmed necrosis and virus-induced inflammation. Cell 137, 1112-1123 (2009).

21. Degterev, A. et al. Chemical inhibitor of nonapoptotic cell death with therapeutic potential for ischemic brain injury. Nat. Chem. Biol. 1, 112-119 (2005).

22. Christofferson, D. E. \& Yuan, J. Necroptosis as an alternative form of programmed cell death. Curr. Opin. Cell Biol. 22, 263-268 (2010).

23. Galluzzi, L. \& Kroemer, G. Necroptosis: a specialized pathway of programmed necrosis. Cell 135, 1161-1163 (2008).

24. Festjens, N., Vanden Berghe, T. \& Vandenabeele, P. Necrosis, a wellorchestrated form of cell demise: signalling cascades, important mediators and concomitant immune response. Biochim. Biophys. Acta 1757, 1371-1387 (2006).

25. Hitomi, J. et al. Identification of a molecular signaling network that regulates a cellular necrotic cell death pathway. Cell 135, 1311-1323 (2008).

26. He, S. et al. Receptor interacting protein kinase-3 determines cellular necrotic response to TNF-[alpha]. Cell 137, 1100-1111 (2009).

27. Vanlangenakker, $\mathrm{N}$. et al. CIAP1 and TAK1 protect cells from TNF-induced necrosis by preventing RIP1/RIP3-dependent reactive oxygen species production. Cell Death Differ. 18, 656-665 (2011).

28. Vandenabeele, P., Declercq, W., Van Herreweghe, F. \& Vanden Berghe, T. The role of the kinases RIP1 and RIP3 in TNF-induced necrosis. Sci. Signal. 3, re4 (2010).

29. Cai, Z. et al. Plasma membrane translocation of trimerized MLKL protein is required for TNF-induced necroptosis. Nat. Cell Biol. 16, 55-65 (2014).

30. McComb, S. et al. Type-I interferon signaling through ISGF3 complex is required for sustained Rip3 activation and necroptosis in macrophages. Proc. Natl Acad. Sci. USA 111, E3206-E3213 (2014).

31. Ofengeim, D. \& Yuan, J. Regulation of RIP1 kinase signalling at the crossroads of inflammation and cell death. Nat. Rev. Mol. Cell Biol. 14, 727-736 (2013).
32. Polykratis, A. et al. Cutting edge: RIPK1 Kinase inactive mice are viable and protected from TNF-induced necroptosis in vivo. J. Immunol. 193, 1539-1543 (2014).

33. Berger, S. B. et al. Cutting Edge: RIP1 kinase activity is dispensable for normal development but is a key regulator of inflammation in SHARPIN-deficient mice. J. Immunol. 192, 5476-5480 (2014).

34. Xie, T. et al. Structural basis of RIP1 inhibition by necrostatins. Structure 21, 493-499 (2013).

35. Takahashi, N. et al. Necrostatin-1 analogues: critical issues on the specificity, activity and in vivo use in experimental disease models. Cell Death Dis. 3, e437 (2012).

36. Mandal, P. et al. RIP3 induces apoptosis independent of pronecrotic kinase activity. Mol. Cell 56, 481-495 (2014).

37. Bertrand, M. J. et al. CIAP1 and CIAP2 facilitate cancer cell survival by functioning as E3 ligases that promote RIP1 ubiquitination. Mol. Cell 30, 689-700 (2008).

38. McComb, S. et al. CIAP1 and CIAP2 limit macrophage necroptosis by inhibiting Rip1 and Rip3 activation. Cell Death Differ. 19, 1791-1801 (2012).

39. McComb, S. et al. Activation of concurrent apoptosis and necroptosis by SMAC mimetics for the treatment of refractory and relapsed ALL. Sci. Transl. Med. 8, 339ra370 (2016).

40. Moquin, D. M., McQuade, T. \& Chan, F. K. CYLD deubiquitinates RIP1 in the TNFalpha-induced necrosome to facilitate kinase activation and programmed necrosis. PLOS ONE 8, e76841 (2013).

41. Shembade, N., Ma, A. \& Harhaj, E. W. Inhibition of NF-kappaB signaling by A20 through disruption of ubiquitin enzyme complexes. Science $\mathbf{3 2 7}, \mathbf{1 1 3 5 - 1 1 3 9}$ (2010).

42. Reiley, W., Zhang, M., Wu, X., Granger, E. \& Sun, S. C. Regulation of the deubiquitinating enzyme CYLD by IkappaB kinase gamma-dependent phosphorylation. Mol. Cell. Biol. 25, 3886-3895 (2005).

43. Sun, S. C. CYLD: a tumor suppressor deubiquitinase regulating NF-kappaB activation and diverse biological processes. Cell Death Differ. 17, 25-34 (2010).

44. Harhaj, E. W. \& Dixit, V. M. Deubiquitinases in the regulation of NF-kappaB signaling. Cell Res. 21, 22-39 (2011).

45. Sakamoto, H. et al. Gliotoxin suppresses NF-kappaB activation by selectively inhibiting linear ubiquitin chain assembly complex (LUBAC). ACS Chem. Biol. 10, 675-681 (2015)

46. Chuang, T. H. \& Ulevitch, R. J. Triad3A, an E3 ubiquitin-protein ligase regulating Toll-like receptors. Nat. Immunol. 5, 495-502 (2004).

47. Fearns, C., Pan, Q., Mathison, J. C. \& Chuang, T. H. Triad3A regulates ubiquitination and proteasomal degradation of RIP1 following disruption of Hsp90 binding. J. Biol. Chem. 281, 34592-34600 (2006).

48. Kaczmarek, A., Vandenabeele, P. \& Krysko, D. V. Necroptosis: the release of damage-associated molecular patterns and its physiological relevance Immunity 38, 209-223 (2013).

49. Vandenabeele, P., Galluzzi, L., Vanden Berghe, T. \& Kroemer, G. Molecular mechanisms of necroptosis: an ordered cellular explosion. Nat. Rev. Mol. Cell Biol. 11, 700-714 (2010).

50. Degterev, A. et al. Identification of RIP1 kinase as a specific cellular target of necrostatins. Nat. Chem. Biol. 4, 313-321 (2008).

51. He, S., Liang, Y., Shao, F. \& Wang, X. Toll-like receptors activate programmed necrosis in macrophages through a receptor-interacting kinase-3-mediated pathway. Proc. Natl Acad. Sci. USA 108, 20054-20059 (2011).

52. Dondelinger, Y. et al. NF-kappaB-independent role of IKKalpha/IKKbeta in preventing RIPK1 kinase-dependent apoptotic and necroptotic cell death during TNF signaling. Mol. Cell 60, 63-76 (2015).

53. Jaco, I. et al. MK2 phosphorylates RIPK1 to prevent TNF-induced cell death. Mol. Cell 66, 698-710 (2017).

54. McQuade, T., Cho, Y. \& Chan, F. K. Positive and negative phosphorylation regulates RIP1- and RIP3-induced programmed necrosis. Biochem. J. 456 409-415 (2013).

55. Cook, W. D. et al. RIPK1- and RIPK3-induced cell death mode is determined by target availability. Cell Death Differ. 21, 1600-1612 (2014).

56. Wu, X. N. et al. Distinct roles of RIP1-RIP3 hetero- and RIP3-RIP3 homointeraction in mediating necroptosis. Cell Death Differ. 21, 1709-1720 (2014).

57. Beug, S. T., Cheung, H. H., LaCasse, E. C. \& Korneluk, R. G. Modulation of immune signalling by inhibitors of apoptosis. Trends Immunol. 33, 535-545 (2012).

58. Peltzer, N., Darding, M., Walczak, H. Holding RIPK1 on the ubiquitin leash in TNFR1 signaling. Trends Cell Biol. 26, 445-461 (2016). 
59. Vanden Berghe, T., Linkermann, A., Jouan-Lanhouet, S., Walczak, H. \& Vandenabeele, P. Regulated necrosis: the expanding network of non-apoptotic cell death pathways. Nat. Rev. Mol. Cell Biol. 15, 135-147 (2014).

60. Kaiser, W. J. et al. RIP3 mediates the embryonic lethality of caspase-8-deficient mice. Nature 471, 368-372 (2011).

61. Dillon, C. P. et al. RIPK1 blocks early postnatal lethality mediated by caspase-8 and RIPK3. Cell 157, 1189-1202 (2014).

62. McComb, S. et al. Cathepsins limit macrophage necroptosis through cleavage of Rip1 kinase. J. Immunol. 192, 5671-5678 (2014).

63. Malladi, S., Challa-Malladi, M., Fearnhead, H. O. \& Bratton, S. B. The Apaf1 *procaspase-9 apoptosome complex functions as a proteolytic-based molecular timer. EMBO J. 28, 1916-1925 (2009).
64. Lawlor, K. E. et al. RIPK3 promotes cell death and NLRP3 inflammasome activation in the absence of MLKL. Nat. Commun. 6, 6282 (2015).

65. Newton, K. et al. RIPK3 deficiency or catalytically inactive RIPK1 provides greater benefit than MLKL deficiency in mouse models of inflammation and tissue injury. Cell Death Differ. 23, 1565-1576 (2016).

66. Voll, R. E. et al. Immunosuppressive effects of apoptotic cells. Nature $\mathbf{3 9 0}$ 350-351 (1997).

67. Pasparakis, M. \& Vandenabeele, P. Necroptosis and its role in inflammation. Nature 517, 311-320 (2015).

68. Reiley, W. W. et al. Regulation of T cell development by the deubiquitinating enzyme CYLD. Nat. Immunol. 7, 411-417 (2006). 